\title{
PRESENCIA DE CARACOLES LYMNAEIDAE CON FORMAS LARVARIAS DE Fasciola hepatica EN ALTITUDES SOBRE LOS 4000 MSNM EN LA SIERRA SUR DEL PERÚ
}

\author{
Presence of Lymnaeidae Snails with Larvae of $\boldsymbol{F}$. hepatica in Altitudes \\ Over 4000 m Above Sea Level in the Southern Highlands of Peru
}

\author{
Pablo Londoñe B. ${ }^{1}$, Amanda Chávez V. ${ }^{1,2}$, Olga Li E. ${ }^{3}$, Francisco Suárez A. ${ }^{4}$ y \\ Danilo Pezo C. . $^{-}$
}

\section{ResUMen}

\begin{abstract}
El objetivo del estudio fue determinar la presencia de formas larvarias de $F$. hepatica y las especies de caracoles hospederos intermediarios de $F$. hepatica en altitudes superiores a 4000 msnm. Se trabajó en tres altitudes (4000 a 4200, 4200 a 4300, y 4300 a 4500 msnm) colectándose 150 caracoles por cada altitud en la época de lluvias (enero-marzo) del 2004. El área pertenece a la Estación Experimental de la Raya, Universidad Nacional de San Antonio Abad del Cusco. Se utilizaron 50 caracoles para la medición de la concha, 50 para observar el aparato reproductor y la rádula a fin de identificar las especies, y 50 para verificar la presencia de formas larvarias de Fasciola hepatica. Se encontró caracoles de la especie Lymnaea viatrix entre 4000 a $4200 \mathrm{msnm}$ y de la especie Pseudosuccinea columella entre 4200 a $4500 \mathrm{msnm}$. El porcentaje de infestación de los caracoles con las formas larvarias de Fasciola hepatica fue de 48, 46 y 36\% a 4000-4200, 4200-4300, y 4300-4500 msnm, respectivamente, demostrando que el parásito puede sobrevivir sobre los 4000 msnm. La relación entre el nivel de infestación y altitud fue inversamente proporcional.
\end{abstract}

Palabras clave: distoma, Lymnaea viatrix, Pseudosuccinea columella, altitud, infestación, formas larvarias

\section{Abstract}

The objective of the study was to determine the presence of $F$. hepatica larvae and the species of snails that are intermediary hosts of $F$. hepatica in altitudes over $4000 \mathrm{~m}$ above the sea level (masl). The study covered three altitudes (4000-4200, 4200-4300, 4300-4500 masl) and was collected 150 snails per each altitude during the rainy season (January-March, 2004). The area is part of La Raya Experimental Research Station of San Antonio de Abad University, Cusco. Fifty snails were used for measuring the shell, 50 for observing the reproductive organs and the radula to identify the species, and 50 to

\footnotetext{
${ }^{1}$ Laboratorio de Microbiología y Parasitología Veterinaria, ${ }^{3}$ Laboratorio de Patología Clínica, ${ }^{4}$ Laboratorio de Medicina Veterinaria Preventiva, ${ }^{5}$ Estación Experimental del Centro de Investigación IVITA-Maranganí, Facultad de Medicina Veterinaria, Universidad Nacional Mayor de San Marcos, Lima

${ }^{2}$ E-mail: achavezvg@gmail.com
} 


\begin{abstract}
observe the larva forms of Fasciola hepatica. Snails found at 4000-4200 masl were of the Lymnaea viatrix species and at 4200-4500 of the Pseudosuccinea columella species. The level of infestation with larva forms of Fasciola hepatica was 48, 46 and $36 \%$ at 4000-4200, 4200-4300, and 4300-4500 masl respectively, and this shows that the parasite can survive over 4000 masl. The relationship between level of infestation and altitude was inversely proportional.
\end{abstract}

Key words: distoma, Lymnaea viatrix, Pseudosuccinea columella, altitude, infestation, larva forms

\section{INTRODUCCIÓN}

La Distomatosis es una de las enfermedades más importantes que afectan la producción ganadera a nivel mundial, principalmente por las pérdidas económicas que ocasiona; y en el Perú, es la segunda enfermedad en importancia. Es producida por la Fasciola hepatica (Leguía y Casas, 1999), afecta a numerosas especies de animales domésticos y silvestres, y constituye un problema de salud pública (Hurtado y Tantaleán, 1998).

La Fasciola hepatica elimina sus huevos por medio de las heces. El miracidio ciliado, al salir del huevo, no es una larva simple; posee glándulas secretoras necesarias para penetrar al hospedador intermediario. Cuando la larva encuentra la especie de Lymnaea adecuada, pierde los cilios y se inicia la "explosión reproductiva" en cadena, es decir, las redias madres existentes dentro del esporocisto son liberadas y de inmediato comienzan a generarse las redias hijas. Este proceso multiplicador y asexual ocurre principalmente en el hepatopáncreas del molusco. Finalmente, salen las cercarias bien diferenciadas y se adhieren a las hojas de los berros acuáticos al secretar un fluido gomoso y elástico que al solidificarse forja la metacercaria infectante (Carrada-Bravo, 2003).

En el Perú, las pérdidas que ocasiona la distomatosis a la ganadería nacional han sido estimadas en cerca de 11 millones de dólares anuales (Rojas, 1990). Así mismo, el Ministerio de Agricultura del Perú (MINAG, 1973) considera que esta enfermedad ocasiona pérdidas por cerca de 170,000 dólares anuales, solamente en camélidos sudamericanos (CSA), por concepto de mermas productivas y decomiso de hígados parasitados.

En el Perú se han descrito tres especies del caracol Lymnaeidae que son naturalmente infectadas con formas larvarias de F. hepatica: Lymnaea viatrix en Cajamarca y valle del Mantaro (Grados e Ibañez, 1971), Lymnaea diaphana en Arequipa (Córdova et al., 1961) y Pseudosuccinea columella en Tingo María (Tantaleán y Huiza, 1976), así como una especie facultativa Lymnaea cousini (Larrea et al., 1994).

Informaciones sobre la presencia de $F$. hepatica en altitudes superiores a los 4000 msnm son escasas. Se ha reportado tasas de infección entre 15 a $56 \%$ en niños y adultos de áreas rurales en la irrigación de Asillo, Azángaro, Puno (Sánchez et al., 1993) que podrían demostrar que el parásito subsiste a a temperaturas muy frías y a grandes alturas. El presente estudio se llevó a cabo para determinar la presencia de formas larvarias de $F$. hepatica y las especies de caracoles hospederos intermediarios de $F$. hepatica en altitudes superiores a $4000 \mathrm{msnm}$.

\section{Materiales y Métodos}

El muestreo se realizó en tres áreas con altitudes de 4000 a 4200, 4200 a 4300 y 4300 a 4500 msnm, ubicadas en la Estación Ex- 


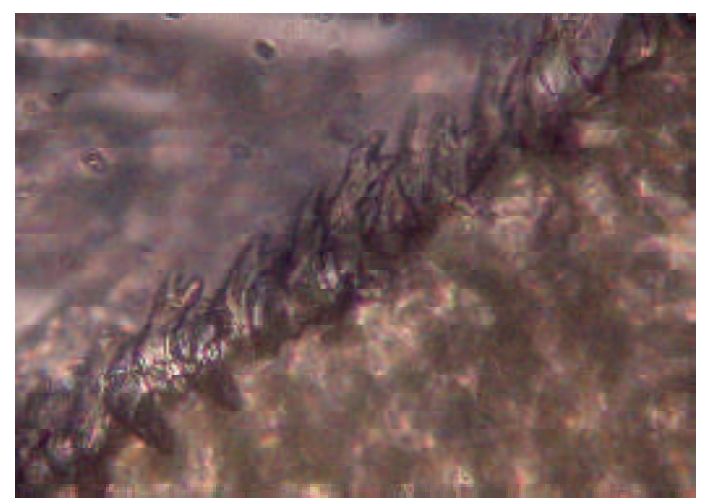

(a)

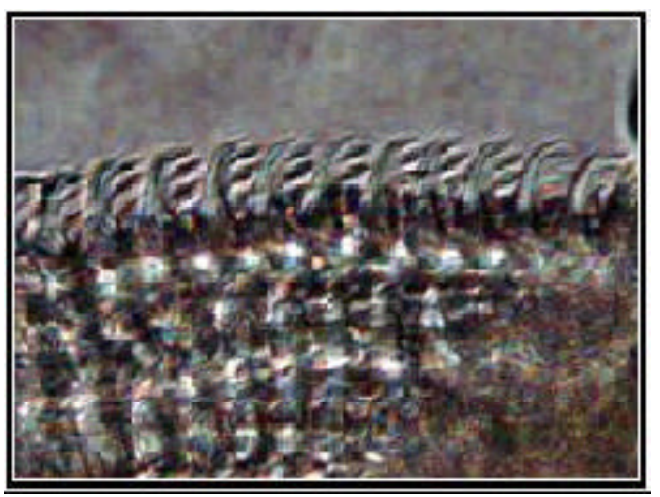

(b)

Figura 1. Vistas de la rádula de los dos tipos de caracoles encontrados entre los 4200 a 4500 msnm, en la Estación Experimental de La Raya- Universidad Nacional San Antonio de Abad, Cusco. (a) Imagen de un caracol Lymnaea viatrix. (b) Imagen de un caracol Pseudosuccinea columella

Cuadro 1. Morfometría de conchas de caracoles encontrados entre los 4200 a $4500 \mathrm{msnm}$, en la Estación Experimental de La Raya, Universidad Nacional San Antonio Abad, Cusco (2004)

\begin{tabular}{lcccccc}
\hline \multirow{2}{*}{ Medidas } & \multicolumn{3}{c}{ Lymnaea viatrix } & \multicolumn{3}{c}{ Pseudosuccinea columella } \\
\cline { 2 - 7 } & Promedio & d.e. & Rango & Promedio & d.e. & Rango \\
\hline Ancho $(\mathrm{mm})$ & 1.1 & 1.0 & $0.8-1.3$ & 3.1 & 1.0 & $0.9-4.7$ \\
Largo $(\mathrm{mm})$ & 4.5 & 0.4 & $3.5-5.0$ & 6.2 & 1.8 & $2.2-8.2$ \\
Circunvoluciones $\left(\mathrm{N}^{\circ}\right)$ & 4.24 & 0.43 & $4.0-5.0$ & 3.0 & 0 & 3.0 \\
Abertura (mm) & 0.7 & 0.2 & $0-5-1.1$ & 2.1 & 0.9 & $0.3-3.5$ \\
\hline
\end{tabular}

Cuadro 2. Índice de infección por formas larvarias de Fasciola hepatica en 50 caracoles por cada altitud entre los 4200 a $4500 \mathrm{msnm}$, en la Estación Experimental de La Raya, Universidad Nacional San Antonio Abad, Cusco (2004)

\begin{tabular}{llccc}
\hline \multirow{2}{*}{$\begin{array}{l}\text { Altitud } \\
(\mathrm{msnm})\end{array}$} & Especie & \multicolumn{3}{c}{ Caracoles positivos a formas larvarias } \\
\cline { 3 - 5 } & & $\mathrm{n}$ & $\%$ & I.C. $^{1}$ \\
\hline $4000-4200$ & Lymnaea viatrix & 24 & 48 & 14 \\
$4200-4300$ & Pseudosuccinea columella & 23 & 46 & 14 \\
$4300-4500$ & Pseudosuccinea columella & 18 & 36 & 13 \\
\hline
\end{tabular}

\footnotetext{
${ }^{1}$ Intervalo de confianza al 95\%
} 
perimental de La Raya, Universidad Nacional San Antonio de Abad, Cusco, entre enero y marzo (época de lluvias) de 2004. La región presenta una temperatura media mensual de $12.1^{\circ} \mathrm{C}$, precipitación total mensual de $144.7 \mathrm{~mm}$ y humedad relativa media mensual de $73.7 \%$ en los meses de enero a marzo. Las muestras se trabajaron en los laboratorios del Centro de Investigación IVITAMaranganí de la Universidad Nacional Mayor de San Marcos, Cusco.

Se colectaron 150 caracoles de la familia Lymnaeidae en cada una de las tres alturas ecológicas. De estos, 50 se emplearon para la identificación externa (concha), 50 para la identificación de la rádula y el aparato reproductor, y 50 para observar las formas larvarias de Fasciola hepatica, según trabajos realizados por Tantaleán et al. (2000).

Los caracoles se colectaron siguiendo el método descrito por Manga-González et al. (1991). En resumen, los lugares de colecta para cada sitio se escogieron en base a los siguientes criterios: a) lugares con gran cantidad de humedad, y sin presencia de agua, y b) lugares con agua estancada o de bajo movimiento de agua. En estos lugares se recorrió una trayectoria circular por el lapso de 15 minutos. Los moluscos se guardaron en bolsas plásticas, incluyendo agua y lodo, si había, y se llevaron al laboratorio para su respectiva identificación.

La identificación se hizo utilizando los criterios diagnósticos basados en la característica de la conchilla, la rádula y el aparato reproductor. En la disección de los caracoles, observación del aparato reproductor y montaje de la rádula se siguió los procedimientos señalados por Paraense (1976) y Malek (1985). En la caracterización se utilizó las claves de Larrea et al. (1994) y Prepelitchi et al. (2003).

En la visualización de las formas larvarias se tomó un caracol sobre un portaobjeto donde se añadió 3 gotas de agua.
Se le comprimió con una pinza de disección y se observó en el microscopio el movimiento y morfología de las formas larvarias (Leguía y Casas, 1999).

En la cuantificación de los resultados se empleó la estadística descriptiva, calculándose como medidas de posición la media aritmética y como medidas de dispersión, el rango y la desviación estándar (Pagano et al., 2001). El nivel de caracoles parasitados por las formas larvarias de la Fasciola hepatica se expresó como frecuencias porcentuales relativas con sus respectivos intervalos de confianza del 95\% mediante la fórmula para una proporción (Daniel, 1996).

\section{Resultados}

Los caracoles colectados entre 4000 a $4200 \mathrm{msnm}$ poseían una fórmula radular de 26-1-26, es decir, un diente central con una cúspide accesoria derecha, 26 dientes laterales triangulares cónicos y generalmente bicúspide a ambos extremos (Fig. 1a), indicativo de Lymnaea viatrix; mientras que los caracoles colectados en las otras dos zonas comprendidas entre los 4200 a 4500 msnm poseían una fórmula radular de 31-1-31, es decir: 1 diente central con una cúspide accesoria al lado izquierdo y 31 dientes laterales tricúspides a ambos extremos (Fig. 1b), indicativo de Pseudosuccinea columella.

Al observar las características del aparato reproductor, se determinó que un grupo de ellos presentaba un saco penial que representaba los 2/3 de la longitud total del prepucio y la próstata presentaba pliegues profundos, compatibles con la especie Lymnaea viatrix. En el otro grupo de caracoles, el saco penial era el $1 / 3$ de la longitud total del prepucio y la próstata no presentaba pliegues, lo cual era compatible con la especie Pseudosuccinea columella .

Los tamaños promedio de algunas características morfológicas de las dos especies de 
caracoles encontrados en el estudio se muestran en el Cuadro 1. Los caracoles de la especie encontrados en las dos altitudes bajo estudio (4200 a 4300 y 4300 a 4500 msnm) tenían medidas similares. En el Cuadro 2 se puede apreciar que el porcentaje de caracoles infectados fue inversamente proporcional a la altitud del hábitat del hospedero intermediario.

\section{Discusión}

Se ha venido sosteniendo que tanto la Fasciola hepatica como sus formas larvarias en los hospederos intermediarios (Lymnaea sp.) no podrían desarrollarse en altitudes superiores a los $4000 \mathrm{msnm}$, donde la temperatura promedio se encuentra por debajo de 10 ${ }^{\circ} \mathrm{C}$ (Soulsby, 1987; Cordero del Campillo et al., 1999); sin embargo, en este estudio se presentan nuevos hallazgos sobre la epidemiología de la distomatosis a esas alturas.

Caracoles pertenecientes a la familia Lymnaeidae fueron hallados en altitudes superiores a los $4000 \mathrm{msnm}$. Las características del sistema radular (Larrea et al., 1994; Hurtado y Tantaleán, 1998), así como las evaluaciones morfológicas del aparato reproductor (Larrea et al., 1993; Prepelitchi et al., 2003) demostraron la presencia de Lymnaea viatrix y Pseudosuccinea columella. Este hallazgo difiere con lo observado por Larrea et al. (1994), quienes no hallaron al caracol Pseudosuccinea columella en la zona de Cusco y Puno, tal vez debido a que estos autores no evaluaron zonas altas como en el presente estudio o probablemente este hallazgo confirma la introducción de esta especie a nuevos hábitats.

Las dimensiones obtenidas para ambas especies fueron muy inferiores a las dimensiones de las conchas obtenidas por Larrea et al. (1993) en la zona de Cusco y Urubamba y por Hurtado y Tantaleán (1998) en la zona de Tacna. Estas variaciones en el tamaño pudieron deberse a la ubicación geográfica y altitud, que determinaría fluctuaciones en el clima. La temperatura obtenida en el lugar de estudio entre enero y febrero varió entre 11.9 y $12.3^{\circ} \mathrm{C}$ y la temperatura mínima media promedio fluctuó entre 4.7 y $6.8{ }^{\circ} \mathrm{C}$ (SENAMHI, 2004), lo que ocasionó que los caracoles se desarrollen en temperaturas cercanas a las condiciones mínimas de requerimiento $\left(10^{\circ} \mathrm{C}\right)$ (Fuentes et al., 1999). Además, es posible que el ecosistema a alturas superiores a los $4000 \mathrm{msnm}$ condicione la disponibilidad de nutrientes para los caracoles, limitando su desarrollo (Oviedo et al., 1995).

El hallazgo de caracoles de la especie Pseudosuccinea columella en altitudes entre los 4200 a 4500 msnm es un indicativo de una mayor adaptabilidad a la altura que el caso del Lymnaea viatrix (Oviedo et al., 1995). Por otro lado, estudios realizados sobre la epidemiología de la distomatosis han demostrado que los caracoles Lymnaeidae tienen una gran capacidad de propagación, al ampliar sus nichos ecológicos a zonas de altura y de medios ambientes adversos (MasComa et al., 1999a). El Perú presenta zonas de gran altitud, donde la densidad del aire y el oxígeno decrecen, siendo bajas la temperatura y la humedad; factores ambientales externos que según Fuentes et al. (1999), influyen negativamente en el desarrollo de las formas larvarias de Fasciola hepatica en los caracoles. En el presente estudio se demuestra lo contrario, pues tanto formas larvarias como el hospedero intermediario de $F$. hepatica logran adaptarse a temperaturas extremas y a altitudes superiores a los 4000 msnm.

El hallazgo de formas larvarias de $F$. hepatica en caracoles de la familia Lymnaeidae en altitudes superiores a los 4000 msnm constituyen evidencias que rectificarían estudios previos (Leguía y Casas, 1999), donde se indicaba que la prevalencia de distomatosis sería baja o nula en altitudes comprendidas entre 4100 a $4800 \mathrm{msnm}$, y con temperaturas medias anuales de $0{ }^{\circ} \mathrm{C}$. Estudios realizados en humanos demuestran que 
existen zonas de alta endemicidad a distomatosis en el altiplano boliviano (Esteban et al., 1999; Fuentes et al., 2001) y en el altiplano peruano (Esteban et al., 2002) con una prevalencia de 15 y $24 \%$, respectivamente. Otra explicación al incremento de áreas distomatósicas se debería al aumento de la carga animal y la movilización de animales de áreas con distoma a zonas libres. Así también, los cambios climáticos a nivel mundial debido al efecto invernadero o calentamiento global estarían contribuyendo al incremento de la temperatura de la zona altoandina beneficiando el desarrollo del caracol y de las formas larvarias de Fasciola hepatica en zonas de mayor altitud.

Oviedo et al. (1995) demostraron que el aislamiento geográfico y la adaptación a grandes altitudes ocasionan alteraciones en la morfología del parásito. Esto sugiere que parásitos como Fasciola hepatica y sus hospederos intermediarios hayan tenido que modificar su estructura para poder sobrevivir en hábitats extremos. Existen reportes de diferencias en el tamaño entre Fasciola hepatica adulta de bovinos versus ovinos y un estudio demuestra que la composicion iónica de la Fasciola hepatica del bovino es diferente a la del ovino (Caseby et al., 1995). Estas diferencias pueden reflejar adaptaciones fisiológicas del parásito a medioambientes diferentes que genéticamente determinan rasgos diferentes. Además, los caracoles de la familia Lymnaeidae están bajo fuerte presión de selección, porque la Fasciola hepatica, al igual que otros tremátodos, disminuyen el potencial reproductivo de sus hospederos intermediarios al parasitarlos. Esta circunstancia eleva la importancia de la evolución, reflejado en el evento de colonización, caracterizado por la peculiar dinámica poblacional de los caracoles dulceacuícolas (Frankham, 1997).

La identificacion de las especies de caracoles presentes en la zona evaluada podría ser validada utilizando técnicas de PCR, ya que los resultados de este estudio demuestran variaciones significativas en las medidas de la concha con respecto a otros estudios (Larrea et al., 1993; Hurtado y Tantaleán, 1998). Existen estudios basados en técnicas de PCR en el altiplano boliviano donde las altitudes y las condiciones medio ambientales son similares, demostrando que el caracol Lymnaea truncatula, originario de Europa, fue introducido a América del Sur (Oviedo et al., 1995; Bargues et al., 1997; JabbourZahab et al., 1997; Mas-Coma et al., 2001), constituyéndose en el hospedero intermediario en regiones altamente endémicas de fascioliasis humana en el altiplano norte boliviano (Esteban et al., 1999; Mas-Coma et al., 1999b).

\section{Conclusiones}

- Los hospederos intermediarios de Fasciola hepatica pueden desarrollarse y hallarse en altitudes comprendidas entre los 4000 a 4500 msnm.

- Los caracoles Lymnaea viatrix y Pseudosuccinea columella se encontraron infectados con formas larvarias de Fasciola hepatica entre los 4000 y $4500 \mathrm{msnm}$.

\section{Literatura Citada}

1. Bargues MD, Mangold AJ, MuñozAntolí C, Pointer JP, Mas-Coma S. 1997. SSU rDNA characterization of lymnaeids transmitting human fascioliasis in South and Central America. J Parasitol 83: 1086-1092.

2. Carrada-Bravo T. 2003. Fascioliasis. Diagnóstico, epidemiología y tratamientos. Rev Gastroenterol Mex 8: 135-142.

3. Caseby RH, Harriot M, Fairweather I. 1995. Ionic composition of the liver fluke Fasciola hepatica from different mammalian hosts and comparasition with host bile. Parasitol Res 81: 394-397. 
4. Cordero del Campillo M, Rojo FA, Sánchez C, Hernández S, Navarrete J, Diaz P, Quiroz H, Carvalho M. 1999. Parasitología veterinaria. Madrid: McGraw-Hill Interamericana. 968 p.

5. Córdova E, Náquira $F$, Náquira C. 1961. Lymnaea diaphana king, como huésped intermediario de Fasciola hepatica en Arequipa. Arch Per Pat Clin 15: 165-172.

6. Daniel WM. 1996. Bioestadística: Base para el análisis de las ciencias de la salud. $5^{\text {ta }}$ ed. México: Ed. Limusa. 878 p.

7. Esteban JG, Flores A, Angles R, MasComa S. 1999. High endemicity of human fascioliasis between Lake Titicaca and La Paz valley, Bolivia. T Roy Soc Trop Med H 93: 151-156.

8. Esteban JG, González C, Bargues MD, Angles R, Sánchez C, Náquira C, Mas-Coma S. 2002. High fascioliasis infection in children linked to a man-made irrigation zone in Perú. Trop Med Int Health 7: 339-348.

9. Frankham R. 1997. Do island populations have less genetic variation than mainland populations? Heredity 78 : 311-327.

10. Fuentes $M V$, Valero MA, Bargues MD, Esteban JG, Angles R, Mas-Coma S. 1999. Analysis of climatic data and forecast indices for human fascioliasis at very high altitude. Ann Trop Med Parasitol 93: 835-850.

11. Fuentes MV, Malone JB, Mas-Coma S. 2001. Validation of a mapping and prediction model for human fasciolosis transmission in Andean very high altitude endemic areas using remote sensing data. Acta Trop 79: 87-95.

12. Grados O, Ibáñez N. 1971. Huésped intermediario de Fasciola hepatica en Cajamarca. Arch Per Pat Clin 25: 185190.

13. Hurtado C, Tantaleán M. 1998. Identificación del huésped intermediario de Fasciola hepatica en la provincia de Candarave, Tacna. Rev Per Parasitol 13: 62-65.
14. Jabbour-Zahab R, Pointier JP, Jourdane J, Jarne P, Oviedo JA, Bargues MD, Mas-Coma S, Angles R, Perera G, Bazan C, Khallaayoune $K$, Renaud F. 1997. Phylogeography and genetic divergence of some lymnaeid snails, intermediate hosts of human and animal fascioliasis, with special reference to lymnaeids from the Bolivian Altiplano. Acta Tropica 64: 191-203.

15. Larrea H, Vivar R, Oviedo ML, Huamán P, Pachas L. 1993. Tres estudios sobre la familia Lymnaeidae, vectores de la fasciolasis en el Perú. Bol Lima 89: 85-96.

16. Larrea H, Oviedo ML, Huamán P. 1994. Descripción anatómica de Lymnaea cousini jousseame, 1887 hospedero potencial de Fasciola hepatica linnaeus, 1758 en algunas localidades del Perú. Bol Lima 91-96: 86-89.

17. Leguía G, Casas E. 1999. Enfermedades parasitarias y atlas parasitológico de camélidos sudamericanos. Lima: Ed de Mar. $190 \mathrm{p}$.

18. Malek E. 1985. Snail host of schistosomiasis and other snailtransmitted diseases in tropical América: A manual. Washington DC: Pan American Health Organization. Scientific Publication No. 478.325 p.

19. Manga-González Y, González-Lanza C, Otero-Merino CB. 1991. Natural infection of Lymnaea truncatula by the liver fluke Fasciola hepatica in the Porma Basin, León, NW Spain. J Helminthol 65: 15-27.

20. Mas-Coma S, Esteban JG, Bargues MD. 1999a. Epidemiology of fascioliasis: a review and proposed new classification. Bull WHO 77: 340-346.

21. Mas-Coma S, Angles R, Esteban JG, Bargues MD, Buchon P, Franken M, Strauss W. 1999b. The northern Bolivian altiplano: A region highly endemic for human fascioliasis. Trop Med Int Health 4: 454-467. 
22. Mas-Coma S, Funatsu IR, Bargues MD. 2001. Fasciola hepatica and lymnaeid snails ocurring at very high altitude in South América. Parasitology 123(Suppl): 115-127.

23. [MINAG] Ministerio de Agricultura. 1973. Estudios de la evaluación de problemas de carne en el Perú. Tomo V. Lima, Perú.

24. Oviedo JA, Bargues MD, Mas-Coma S. 1995. Lymnaeid snails in the human fascioliasis high endemic zone of the Northern Bolivian Altiplano. Res Rev Parasitol 55: 35-43.

25. Pagano M, Gauvreau K, Yescas JM. 2001. Fundamentos de bioestadística. $2^{\text {da }}$ ed. México: Thomson Learning. $525 \mathrm{p}$.

26. Paraense WL. 1976. Lymnaea viatrix: a study of topotypic specimens (Mollusca: Lymnaeidae). Rev Brasil Biol 36: 419-428.

27. Prepelitchi L, Kleiman F, Pietrokovsky S, Moriena R, Racioppi O, Álvarez J, Wisnivesky-Colli C. 2003. First report of Lymnaea columella say, 1817 (Pulmonata: Lymnaeidae) naturally infected with Fasciola hepatica (Linnaeus, 1758)
(Trematoda: Digenea) in Argentina. Mem Inst. Oswaldo Cruz 98: 889-891.

28. Rojas CM. 1990. Parasitismo de los rumiantes domésticos. Terapia, prevención y modelos para su aprendizaje. Lima: MAIJOSA. 324 p.

29. Sánchez C, Aparicio W, Hurtado C. 1993. Distomatosis hepática en la población humana de la Irrigación de AsilloAzángaro (Puno). Res XI Cong Latinoam Parasitol. Lima, Perú.

30. SENAMHI. 2004. Parámetros meteorológicos de la estación Sicuani-CuzcoPerú. Oficina General de Estadística e Informática. Oficio $\mathrm{N}^{\circ} 836$.

31. Soulsby EJ. 1987. Parasitología y enfermedades parasitarias en los animales domésticos. $7^{\mathrm{a}}$ ed. México: $\mathrm{Ed}$ Interamericana. $823 \mathrm{p}$.

32. Tantaleán M, Huiza A. 1976. Los hospederos intermediarios de Fasciola hepatica en el Perú. Infección experimental de Lymnaea columella say. Biota 11(86): 34-37.

33. Tantaleán M, Arroyo L, Miranda E. 2000. Lymnaea Columella Say (Pseudosuccinea columella) como huésped intermediario de Fasciola hepatica en el Perú. Rev Per Parasitol 15: 33-35. 